Ann. Funct. Anal. 7 (2016), no. 2, 303-313

http://dx.doi.org/10.1215/20088752-3505953

ISSN: 2008-8752 (electronic)

ANNALS of

FUNCTIONAL

http://projecteuclid.org/afa

ANALYSIS

\title{
EXTENSION OF THE BEST POLYNOMIAL APPROXIMATION OPERATOR IN VARIABLE EXPONENT LEBESGUE SPACES
}

TENGIZ KOPALIANI, NINO SAMASHVILI, and SHALVA ZVIADADZE*

Communicated by J. Soria

Abstract. Let $\Omega$ be a bounded measurable set in $\mathbb{R}^{n}$. The best polynomial approximation operator was recently extended by Cuenya from $L^{p}$ to $L^{p-1}$.

In this paper, we extend the operator of the best polynomial approximation from $L^{p(\cdot)}(\Omega)$ to the space $L^{p(\cdot)-1}(\Omega)$.

\section{INTRODUCTION}

Let $\Omega$ be a bounded measurable set in $\mathbb{R}^{n}$. Given a measurable function $p$ : $\Omega \longrightarrow(0,+\infty), L^{p(\cdot)}(\Omega)$ denotes the set of measurable functions $f$ on $\Omega$ such that, for some positive $\lambda>0$,

$$
\int_{\Omega}\left(\frac{|f(x)|}{\lambda}\right)^{p(x)} d x<+\infty .
$$

If $1 \leq p(x)<\infty$, then this set becomes a Banach function space when equipped with the norm

$$
\|f\|_{p(\cdot)}=\inf \left\{\lambda>0: \int_{\Omega}\left(\frac{|f(x)|}{\lambda}\right)^{p(x)} d x \leq 1\right\} .
$$

Assume that

$$
p_{-}=\operatorname{ess}_{x \in \mathbb{R}^{n}} p(x), \quad p_{+}=\operatorname{ess}_{x \in \mathbb{R}^{n}} p(x)
$$

Copyright 2016 by the Tusi Mathematical Research Group.

Received Apr. 28, 2015; Accepted Aug. 16, 2015.

${ }^{*}$ Corresponding author.

2010 Mathematics Subject Classification. Primary 41A50; Secondary 41A52, 41A10, 42A10.

Keywords. variable exponent Lebesgue spaces, the best polynomial approximation, extension of the operator. 
Ann. Funct. Anal. 7 (2016), no. 2, 303-313

http://dx.doi.org/10.1215/20088752-3505953

ISSN: 2008-8752 (electronic)

ANNALS of

FUNCTIONAL

http://projecteuclid.org/afa

ANALYSIS

\section{EXTENSION OF THE BEST POLYNOMIAL APPROXIMATION OPERATOR IN VARIABLE EXPONENT LEBESGUE SPACES}

TENGIZ KOPALIANI, NINO SAMASHVILI, and SHALVA ZVIADADZE*

Communicated by J. Soria

Abstract. Let $\Omega$ be a bounded measurable set in $\mathbb{R}^{n}$. The best polynomial approximation operator was recently extended by Cuenya from $L^{p}$ to $L^{p-1}$.

In this paper, we extend the operator of the best polynomial approximation from $L^{p(\cdot)}(\Omega)$ to the space $L^{p(\cdot)-1}(\Omega)$.

\section{INTRODUCTION}

Let $\Omega$ be a bounded measurable set in $\mathbb{R}^{n}$. Given a measurable function $p$ : $\Omega \longrightarrow(0,+\infty), L^{p(\cdot)}(\Omega)$ denotes the set of measurable functions $f$ on $\Omega$ such that, for some positive $\lambda>0$,

$$
\int_{\Omega}\left(\frac{|f(x)|}{\lambda}\right)^{p(x)} d x<+\infty .
$$

If $1 \leq p(x)<\infty$, then this set becomes a Banach function space when equipped with the norm

$$
\|f\|_{p(\cdot)}=\inf \left\{\lambda>0: \int_{\Omega}\left(\frac{|f(x)|}{\lambda}\right)^{p(x)} d x \leq 1\right\} .
$$

Assume that

$$
p_{-}=\operatorname{ess}_{x \in \mathbb{R}^{n}} p(x), \quad p_{+}=\operatorname{ess}_{x \in \mathbb{R}^{n}} p(x)
$$

Copyright 2016 by the Tusi Mathematical Research Group.

Received Apr. 28, 2015; Accepted Aug. 16, 2015.

${ }^{*}$ Corresponding author.

2010 Mathematics Subject Classification. Primary 41A50; Secondary 41A52, 41A10, 42A10.

Keywords. variable exponent Lebesgue spaces, the best polynomial approximation, extension of the operator. 
Ann. Funct. Anal. 7 (2016), no. 2, 303-313

http://dx.doi.org/10.1215/20088752-3505953

ISSN: 2008-8752 (electronic)

ANNALS of

FUNCTIONAL

http://projecteuclid.org/afa

ANALYSIS

\section{EXTENSION OF THE BEST POLYNOMIAL APPROXIMATION OPERATOR IN VARIABLE EXPONENT LEBESGUE SPACES}

TENGIZ KOPALIANI, NINO SAMASHVILI, and SHALVA ZVIADADZE*

Communicated by J. Soria

Abstract. Let $\Omega$ be a bounded measurable set in $\mathbb{R}^{n}$. The best polynomial approximation operator was recently extended by Cuenya from $L^{p}$ to $L^{p-1}$.

In this paper, we extend the operator of the best polynomial approximation from $L^{p(\cdot)}(\Omega)$ to the space $L^{p(\cdot)-1}(\Omega)$.

\section{INTRODUCTION}

Let $\Omega$ be a bounded measurable set in $\mathbb{R}^{n}$. Given a measurable function $p$ : $\Omega \longrightarrow(0,+\infty), L^{p(\cdot)}(\Omega)$ denotes the set of measurable functions $f$ on $\Omega$ such that, for some positive $\lambda>0$,

$$
\int_{\Omega}\left(\frac{|f(x)|}{\lambda}\right)^{p(x)} d x<+\infty .
$$

If $1 \leq p(x)<\infty$, then this set becomes a Banach function space when equipped with the norm

$$
\|f\|_{p(\cdot)}=\inf \left\{\lambda>0: \int_{\Omega}\left(\frac{|f(x)|}{\lambda}\right)^{p(x)} d x \leq 1\right\} .
$$

Assume that

$$
p_{-}=\operatorname{ess}_{x \in \mathbb{R}^{n}} p(x), \quad p_{+}=\operatorname{ess}_{x \in \mathbb{R}^{n}} p(x)
$$

Copyright 2016 by the Tusi Mathematical Research Group.

Received Apr. 28, 2015; Accepted Aug. 16, 2015.

${ }^{*}$ Corresponding author.

2010 Mathematics Subject Classification. Primary 41A50; Secondary 41A52, 41A10, 42A10.

Keywords. variable exponent Lebesgue spaces, the best polynomial approximation, extension of the operator. 
Ann. Funct. Anal. 7 (2016), no. 2, 303-313

http://dx.doi.org/10.1215/20088752-3505953

ISSN: 2008-8752 (electronic)

ANNALS of

FUNCTIONAL

http://projecteuclid.org/afa

ANALYSIS

\section{EXTENSION OF THE BEST POLYNOMIAL APPROXIMATION OPERATOR IN VARIABLE EXPONENT LEBESGUE SPACES}

TENGIZ KOPALIANI, NINO SAMASHVILI, and SHALVA ZVIADADZE*

Communicated by J. Soria

Abstract. Let $\Omega$ be a bounded measurable set in $\mathbb{R}^{n}$. The best polynomial approximation operator was recently extended by Cuenya from $L^{p}$ to $L^{p-1}$.

In this paper, we extend the operator of the best polynomial approximation from $L^{p(\cdot)}(\Omega)$ to the space $L^{p(\cdot)-1}(\Omega)$.

\section{INTRODUCTION}

Let $\Omega$ be a bounded measurable set in $\mathbb{R}^{n}$. Given a measurable function $p$ : $\Omega \longrightarrow(0,+\infty), L^{p(\cdot)}(\Omega)$ denotes the set of measurable functions $f$ on $\Omega$ such that, for some positive $\lambda>0$,

$$
\int_{\Omega}\left(\frac{|f(x)|}{\lambda}\right)^{p(x)} d x<+\infty .
$$

If $1 \leq p(x)<\infty$, then this set becomes a Banach function space when equipped with the norm

$$
\|f\|_{p(\cdot)}=\inf \left\{\lambda>0: \int_{\Omega}\left(\frac{|f(x)|}{\lambda}\right)^{p(x)} d x \leq 1\right\} .
$$

Assume that

$$
p_{-}=\operatorname{ess}_{x \in \mathbb{R}^{n}} p(x), \quad p_{+}=\operatorname{ess}_{x \in \mathbb{R}^{n}} p(x)
$$

Copyright 2016 by the Tusi Mathematical Research Group.

Received Apr. 28, 2015; Accepted Aug. 16, 2015.

${ }^{*}$ Corresponding author.

2010 Mathematics Subject Classification. Primary 41A50; Secondary 41A52, 41A10, 42A10.

Keywords. variable exponent Lebesgue spaces, the best polynomial approximation, extension of the operator. 
Ann. Funct. Anal. 7 (2016), no. 2, 303-313

http://dx.doi.org/10.1215/20088752-3505953

ISSN: 2008-8752 (electronic)

ANNALS of

FUNCTIONAL

http://projecteuclid.org/afa

ANALYSIS

\section{EXTENSION OF THE BEST POLYNOMIAL APPROXIMATION OPERATOR IN VARIABLE EXPONENT LEBESGUE SPACES}

TENGIZ KOPALIANI, NINO SAMASHVILI, and SHALVA ZVIADADZE*

Communicated by J. Soria

Abstract. Let $\Omega$ be a bounded measurable set in $\mathbb{R}^{n}$. The best polynomial approximation operator was recently extended by Cuenya from $L^{p}$ to $L^{p-1}$.

In this paper, we extend the operator of the best polynomial approximation from $L^{p(\cdot)}(\Omega)$ to the space $L^{p(\cdot)-1}(\Omega)$.

\section{INTRODUCTION}

Let $\Omega$ be a bounded measurable set in $\mathbb{R}^{n}$. Given a measurable function $p$ : $\Omega \longrightarrow(0,+\infty), L^{p(\cdot)}(\Omega)$ denotes the set of measurable functions $f$ on $\Omega$ such that, for some positive $\lambda>0$,

$$
\int_{\Omega}\left(\frac{|f(x)|}{\lambda}\right)^{p(x)} d x<+\infty .
$$

If $1 \leq p(x)<\infty$, then this set becomes a Banach function space when equipped with the norm

$$
\|f\|_{p(\cdot)}=\inf \left\{\lambda>0: \int_{\Omega}\left(\frac{|f(x)|}{\lambda}\right)^{p(x)} d x \leq 1\right\} .
$$

Assume that

$$
p_{-}=\operatorname{ess}_{x \in \mathbb{R}^{n}} p(x), \quad p_{+}=\operatorname{ess}_{x \in \mathbb{R}^{n}} p(x)
$$

Copyright 2016 by the Tusi Mathematical Research Group.

Received Apr. 28, 2015; Accepted Aug. 16, 2015.

${ }^{*}$ Corresponding author.

2010 Mathematics Subject Classification. Primary 41A50; Secondary 41A52, 41A10, 42A10.

Keywords. variable exponent Lebesgue spaces, the best polynomial approximation, extension of the operator. 
Ann. Funct. Anal. 7 (2016), no. 2, 303-313

http://dx.doi.org/10.1215/20088752-3505953

ISSN: 2008-8752 (electronic)

ANNALS of

FUNCTIONAL

http://projecteuclid.org/afa

ANALYSIS

\section{EXTENSION OF THE BEST POLYNOMIAL APPROXIMATION OPERATOR IN VARIABLE EXPONENT LEBESGUE SPACES}

TENGIZ KOPALIANI, NINO SAMASHVILI, and SHALVA ZVIADADZE*

Communicated by J. Soria

Abstract. Let $\Omega$ be a bounded measurable set in $\mathbb{R}^{n}$. The best polynomial approximation operator was recently extended by Cuenya from $L^{p}$ to $L^{p-1}$.

In this paper, we extend the operator of the best polynomial approximation from $L^{p(\cdot)}(\Omega)$ to the space $L^{p(\cdot)-1}(\Omega)$.

\section{INTRODUCTION}

Let $\Omega$ be a bounded measurable set in $\mathbb{R}^{n}$. Given a measurable function $p$ : $\Omega \longrightarrow(0,+\infty), L^{p(\cdot)}(\Omega)$ denotes the set of measurable functions $f$ on $\Omega$ such that, for some positive $\lambda>0$,

$$
\int_{\Omega}\left(\frac{|f(x)|}{\lambda}\right)^{p(x)} d x<+\infty .
$$

If $1 \leq p(x)<\infty$, then this set becomes a Banach function space when equipped with the norm

$$
\|f\|_{p(\cdot)}=\inf \left\{\lambda>0: \int_{\Omega}\left(\frac{|f(x)|}{\lambda}\right)^{p(x)} d x \leq 1\right\} .
$$

Assume that

$$
p_{-}=\operatorname{ess}_{x \in \mathbb{R}^{n}} p(x), \quad p_{+}=\operatorname{ess}_{x \in \mathbb{R}^{n}} p(x)
$$

Copyright 2016 by the Tusi Mathematical Research Group.

Received Apr. 28, 2015; Accepted Aug. 16, 2015.

${ }^{*}$ Corresponding author.

2010 Mathematics Subject Classification. Primary 41A50; Secondary 41A52, 41A10, 42A10.

Keywords. variable exponent Lebesgue spaces, the best polynomial approximation, extension of the operator. 
Ann. Funct. Anal. 7 (2016), no. 2, 303-313

http://dx.doi.org/10.1215/20088752-3505953

ISSN: 2008-8752 (electronic)

ANNALS of

FUNCTIONAL

http://projecteuclid.org/afa

ANALYSIS

\section{EXTENSION OF THE BEST POLYNOMIAL APPROXIMATION OPERATOR IN VARIABLE EXPONENT LEBESGUE SPACES}

TENGIZ KOPALIANI, NINO SAMASHVILI, and SHALVA ZVIADADZE*

Communicated by J. Soria

Abstract. Let $\Omega$ be a bounded measurable set in $\mathbb{R}^{n}$. The best polynomial approximation operator was recently extended by Cuenya from $L^{p}$ to $L^{p-1}$.

In this paper, we extend the operator of the best polynomial approximation from $L^{p(\cdot)}(\Omega)$ to the space $L^{p(\cdot)-1}(\Omega)$.

\section{INTRODUCTION}

Let $\Omega$ be a bounded measurable set in $\mathbb{R}^{n}$. Given a measurable function $p$ : $\Omega \longrightarrow(0,+\infty), L^{p(\cdot)}(\Omega)$ denotes the set of measurable functions $f$ on $\Omega$ such that, for some positive $\lambda>0$,

$$
\int_{\Omega}\left(\frac{|f(x)|}{\lambda}\right)^{p(x)} d x<+\infty .
$$

If $1 \leq p(x)<\infty$, then this set becomes a Banach function space when equipped with the norm

$$
\|f\|_{p(\cdot)}=\inf \left\{\lambda>0: \int_{\Omega}\left(\frac{|f(x)|}{\lambda}\right)^{p(x)} d x \leq 1\right\} .
$$

Assume that

$$
p_{-}=\operatorname{ess}_{x \in \mathbb{R}^{n}} p(x), \quad p_{+}=\operatorname{ess}_{x \in \mathbb{R}^{n}} p(x)
$$

Copyright 2016 by the Tusi Mathematical Research Group.

Received Apr. 28, 2015; Accepted Aug. 16, 2015.

${ }^{*}$ Corresponding author.

2010 Mathematics Subject Classification. Primary 41A50; Secondary 41A52, 41A10, 42A10.

Keywords. variable exponent Lebesgue spaces, the best polynomial approximation, extension of the operator. 
Ann. Funct. Anal. 7 (2016), no. 2, 303-313

http://dx.doi.org/10.1215/20088752-3505953

ISSN: 2008-8752 (electronic)

ANNALS of

FUNCTIONAL

http://projecteuclid.org/afa

ANALYSIS

\section{EXTENSION OF THE BEST POLYNOMIAL APPROXIMATION OPERATOR IN VARIABLE EXPONENT LEBESGUE SPACES}

TENGIZ KOPALIANI, NINO SAMASHVILI, and SHALVA ZVIADADZE*

Communicated by J. Soria

Abstract. Let $\Omega$ be a bounded measurable set in $\mathbb{R}^{n}$. The best polynomial approximation operator was recently extended by Cuenya from $L^{p}$ to $L^{p-1}$.

In this paper, we extend the operator of the best polynomial approximation from $L^{p(\cdot)}(\Omega)$ to the space $L^{p(\cdot)-1}(\Omega)$.

\section{INTRODUCTION}

Let $\Omega$ be a bounded measurable set in $\mathbb{R}^{n}$. Given a measurable function $p$ : $\Omega \longrightarrow(0,+\infty), L^{p(\cdot)}(\Omega)$ denotes the set of measurable functions $f$ on $\Omega$ such that, for some positive $\lambda>0$,

$$
\int_{\Omega}\left(\frac{|f(x)|}{\lambda}\right)^{p(x)} d x<+\infty .
$$

If $1 \leq p(x)<\infty$, then this set becomes a Banach function space when equipped with the norm

$$
\|f\|_{p(\cdot)}=\inf \left\{\lambda>0: \int_{\Omega}\left(\frac{|f(x)|}{\lambda}\right)^{p(x)} d x \leq 1\right\} .
$$

Assume that

$$
p_{-}=\operatorname{ess}_{x \in \mathbb{R}^{n}} p(x), \quad p_{+}=\operatorname{ess}_{x \in \mathbb{R}^{n}} p(x)
$$

Copyright 2016 by the Tusi Mathematical Research Group.

Received Apr. 28, 2015; Accepted Aug. 16, 2015.

${ }^{*}$ Corresponding author.

2010 Mathematics Subject Classification. Primary 41A50; Secondary 41A52, 41A10, 42A10.

Keywords. variable exponent Lebesgue spaces, the best polynomial approximation, extension of the operator. 
Ann. Funct. Anal. 7 (2016), no. 2, 303-313

http://dx.doi.org/10.1215/20088752-3505953

ISSN: 2008-8752 (electronic)

ANNALS of

FUNCTIONAL

http://projecteuclid.org/afa

ANALYSIS

\section{EXTENSION OF THE BEST POLYNOMIAL APPROXIMATION OPERATOR IN VARIABLE EXPONENT LEBESGUE SPACES}

TENGIZ KOPALIANI, NINO SAMASHVILI, and SHALVA ZVIADADZE*

Communicated by J. Soria

Abstract. Let $\Omega$ be a bounded measurable set in $\mathbb{R}^{n}$. The best polynomial approximation operator was recently extended by Cuenya from $L^{p}$ to $L^{p-1}$.

In this paper, we extend the operator of the best polynomial approximation from $L^{p(\cdot)}(\Omega)$ to the space $L^{p(\cdot)-1}(\Omega)$.

\section{INTRODUCTION}

Let $\Omega$ be a bounded measurable set in $\mathbb{R}^{n}$. Given a measurable function $p$ : $\Omega \longrightarrow(0,+\infty), L^{p(\cdot)}(\Omega)$ denotes the set of measurable functions $f$ on $\Omega$ such that, for some positive $\lambda>0$,

$$
\int_{\Omega}\left(\frac{|f(x)|}{\lambda}\right)^{p(x)} d x<+\infty .
$$

If $1 \leq p(x)<\infty$, then this set becomes a Banach function space when equipped with the norm

$$
\|f\|_{p(\cdot)}=\inf \left\{\lambda>0: \int_{\Omega}\left(\frac{|f(x)|}{\lambda}\right)^{p(x)} d x \leq 1\right\} .
$$

Assume that

$$
p_{-}=\operatorname{ess}_{x \in \mathbb{R}^{n}} p(x), \quad p_{+}=\operatorname{ess}_{x \in \mathbb{R}^{n}} p(x)
$$

Copyright 2016 by the Tusi Mathematical Research Group.

Received Apr. 28, 2015; Accepted Aug. 16, 2015.

${ }^{*}$ Corresponding author.

2010 Mathematics Subject Classification. Primary 41A50; Secondary 41A52, 41A10, 42A10.

Keywords. variable exponent Lebesgue spaces, the best polynomial approximation, extension of the operator. 
Ann. Funct. Anal. 7 (2016), no. 2, 303-313

http://dx.doi.org/10.1215/20088752-3505953

ISSN: 2008-8752 (electronic)

ANNALS of

FUNCTIONAL

http://projecteuclid.org/afa

ANALYSIS

\section{EXTENSION OF THE BEST POLYNOMIAL APPROXIMATION OPERATOR IN VARIABLE EXPONENT LEBESGUE SPACES}

TENGIZ KOPALIANI, NINO SAMASHVILI, and SHALVA ZVIADADZE*

Communicated by J. Soria

Abstract. Let $\Omega$ be a bounded measurable set in $\mathbb{R}^{n}$. The best polynomial approximation operator was recently extended by Cuenya from $L^{p}$ to $L^{p-1}$.

In this paper, we extend the operator of the best polynomial approximation from $L^{p(\cdot)}(\Omega)$ to the space $L^{p(\cdot)-1}(\Omega)$.

\section{INTRODUCTION}

Let $\Omega$ be a bounded measurable set in $\mathbb{R}^{n}$. Given a measurable function $p$ : $\Omega \longrightarrow(0,+\infty), L^{p(\cdot)}(\Omega)$ denotes the set of measurable functions $f$ on $\Omega$ such that, for some positive $\lambda>0$,

$$
\int_{\Omega}\left(\frac{|f(x)|}{\lambda}\right)^{p(x)} d x<+\infty .
$$

If $1 \leq p(x)<\infty$, then this set becomes a Banach function space when equipped with the norm

$$
\|f\|_{p(\cdot)}=\inf \left\{\lambda>0: \int_{\Omega}\left(\frac{|f(x)|}{\lambda}\right)^{p(x)} d x \leq 1\right\} .
$$

Assume that

$$
p_{-}=\operatorname{ess}_{x \in \mathbb{R}^{n}} p(x), \quad p_{+}=\operatorname{ess}_{x \in \mathbb{R}^{n}} p(x)
$$

Copyright 2016 by the Tusi Mathematical Research Group.

Received Apr. 28, 2015; Accepted Aug. 16, 2015.

${ }^{*}$ Corresponding author.

2010 Mathematics Subject Classification. Primary 41A50; Secondary 41A52, 41A10, 42A10.

Keywords. variable exponent Lebesgue spaces, the best polynomial approximation, extension of the operator. 
Ann. Funct. Anal. 7 (2016), no. 2, 303-313

http://dx.doi.org/10.1215/20088752-3505953

ISSN: 2008-8752 (electronic)

ANNALS of

FUNCTIONAL

http://projecteuclid.org/afa

ANALYSIS

\section{EXTENSION OF THE BEST POLYNOMIAL APPROXIMATION OPERATOR IN VARIABLE EXPONENT LEBESGUE SPACES}

TENGIZ KOPALIANI, NINO SAMASHVILI, and SHALVA ZVIADADZE*

Communicated by J. Soria

Abstract. Let $\Omega$ be a bounded measurable set in $\mathbb{R}^{n}$. The best polynomial approximation operator was recently extended by Cuenya from $L^{p}$ to $L^{p-1}$.

In this paper, we extend the operator of the best polynomial approximation from $L^{p(\cdot)}(\Omega)$ to the space $L^{p(\cdot)-1}(\Omega)$.

\section{INTRODUCTION}

Let $\Omega$ be a bounded measurable set in $\mathbb{R}^{n}$. Given a measurable function $p$ : $\Omega \longrightarrow(0,+\infty), L^{p(\cdot)}(\Omega)$ denotes the set of measurable functions $f$ on $\Omega$ such that, for some positive $\lambda>0$,

$$
\int_{\Omega}\left(\frac{|f(x)|}{\lambda}\right)^{p(x)} d x<+\infty .
$$

If $1 \leq p(x)<\infty$, then this set becomes a Banach function space when equipped with the norm

$$
\|f\|_{p(\cdot)}=\inf \left\{\lambda>0: \int_{\Omega}\left(\frac{|f(x)|}{\lambda}\right)^{p(x)} d x \leq 1\right\} .
$$

Assume that

$$
p_{-}=\operatorname{ess}_{x \in \mathbb{R}^{n}} p(x), \quad p_{+}=\operatorname{ess}_{x \in \mathbb{R}^{n}} p(x)
$$

Copyright 2016 by the Tusi Mathematical Research Group.

Received Apr. 28, 2015; Accepted Aug. 16, 2015.

${ }^{*}$ Corresponding author.

2010 Mathematics Subject Classification. Primary 41A50; Secondary 41A52, 41A10, 42A10.

Keywords. variable exponent Lebesgue spaces, the best polynomial approximation, extension of the operator. 